\title{
Propuesta de un biodigestor tubular de polietileno para producir biogás a partir de paja de arroz y agua del río Utcubamba en la ciudad de Bagua Grande -
} Amazonas, 2016.

\section{Proposal of a tubular polyethylene biodigester to produce biogas from rice straw and water of the Utcubamba river in the city of Bagua Grande -Amazonas, 2016.}

Carlos Miguel Campos - Peralta ${ }^{1}$

\section{RESUMEN}

Se diseñó un biodigestor tubular de polietileno para obtener biogás a partir de paja de arroz con agua del río Utcubamba, para sustituir el uso de leña de una familia promedio. Se construyó tres biodigestores experimentales para producir biogás y estimar la producción de biogás a partir de paja de arroz y agua del río Utcubamba, El experimento se realizó bajo condiciones ambientales no controladas, a una proporción biomasa - agua de 1:2 (2kg de substrato y $4 \mathrm{~L}$ de agua). El volumen de biogás se obtuvo, utilizando un manómetro de tubo en U y la ley de gases ideales. Los datos se analizaron con el programa Statistics 23.0, mediante una prueba de comparación de medias. Se concluyó que el volumen de biogás generado es 541 $\mathrm{ml}$ en época seca y $534 \mathrm{ml}$ en época húmeda. En base a los resultados obtenidos se diseñó un biodigestor tubular de polietileno de $5,23 \mathrm{~m}^{3}$ (7,69 $\mathrm{m}^{3}$ de biogás diariamente), de bajo costo (1 174,90 soles), con un retorno de la inversión inicial en menos de 4 años, lo que contribuirá a sustituir el consumo de leña de una familia promedio y a reducir la contaminación ambiental generada en Bagua Grande - Amazonas.

Palabras clave: biogás, paja de arroz, biodigestor tubular.

\begin{abstract}
ABSTRAC
A tubular biodigester of polyethylene was designed to obtain biogas from rice straw with water from the Utcubamba River, to replace the use of firewood from an average family. Three experimental biodigesters were constructed to produce biogas and to estimate the biogas production from rice straw and Utcubamba river water. The experiment was carried out under uncontrolled environmental conditions at biomass water ratio of $1: 2$ ( $2 \mathrm{~kg}$ of substrate And $4 \mathrm{~L}$ of water). The volume of biogas was obtained using a U-tube manometer and the ideal gas law. The data were analyzed using the Statistics 23.0 program, using a means comparison test. It was concluded that the volume of biogas generated is $541 \mathrm{ml}$ in the dry season and 534 $\mathrm{ml}$ in the wet season. Based on the results obtained, a tubular biodigester of polyethylene of $5,23 \mathrm{~m}^{3}(7,69$ $\mathrm{m} 3$ of biogas daily), of low cost (1 174.90 soles) was designed, with a return of the initial investment in less than 4 Years, which will contribute to substitute the consumption of firewood of an average family and to reduce the environmental pollution generated in Bagua Grande - Amazonas.
\end{abstract}

Key words: biogas, rice straw, tubular biodigestor.

\footnotetext{
${ }^{1}$ Bachiller en Ingeniería Ambiental, egresado de la Universidad Nacional Toribio Rodríguez de Mendoza, correo electrónico: campos_10@hotmail.com
} 


\section{INTRODUCCIÓN}

Gutiérrez et al, (2012) afirma que "resulta sumamente importante hacer investigación en el campo de los biocombustibles para obtener mejores rendimientos y calidad en cuanto a su producción reduciendo a la vez la contaminación ambiental cuando se utilizan residuos como materia prima" En este sentido, el biogás, (...) se considera una forma de energía limpia y renovable que puede sustituir fuentes de energía convencionales, especialmente en áreas rurales. (Weiland, 2010, citado en Contreras et al, 2012). "La cosecha y producción de arroz genera biomasa residual que actualmente no posee una ruta de disposición ambientalmente sostenible; sin embargo podría ser una fuente importante de carbono para procesos energéticos" (Contreras et al., 2012). En Bagua Grande, el arroz es uno de los principales cultivos, el cual genera una gran cantidad de residuos agrícolas (paja, cascara y residuos del proceso de secado), los cuales al ser quemados o descompuestos al aire libre, generan emisiones de gases de efecto invernadero (GEI) que contaminan el medio ambiente. García (2010) afirma que "cada hectárea de arroz genera 4.86 toneladas de paja de arroz". Según un informe del Panel Intergubernamental sobre el Cambio Climático (IPCC, 2007), el 30\% de las emisiones globales de metano antropogénico y el $11 \%$ de óxido nitroso provienen de campos de arroz. Su et al. (2015), afirman que el metano $\left(\mathrm{CH}_{4}\right)$ "es responsable de aproximadamente el $20 \%$ del efecto de calentamiento global, siendo los arrozales la mayor fuente de metano antropogénico (...) con emisiones anuales de 25 a 100 millones toneladas" Esto ocasiona severos impactos al medio ambiente y a la salud de las personas.
Bagua Grande es afectada además por la escasez de agua de buena calidad para consumo humano, a pesar de la cercanía al río Utcubamba, cuyas aguas no son aptas para consumo humano.

En Cuba se concluyó que "el potencial de biogás de $0,52 \mathrm{~m}^{3} \mathrm{kgSV}^{-1}$ correspondió a la paja de arroz como mono sustrato durante un tiempo de digestión de 36 días, bajo condiciones termofílicas" (Contreras et al., 2012)". Chandra et $a l$, (citado en Contreras et al, 2012) "reportan para la paja un rendimiento de metano $167 \mathrm{~kg} \mathrm{CH}_{4} \mathrm{~T}^{-}$ 1”. Según Ferrer et al., (2009), en los proyectos piloto que se presentan, ubicados en Perú, se han implementado alrededor de 20 biodigestores familiares, en comunidades rurales de la zona de Cusco y de Cajamarca. En general se trata de biodigestores tubulares de polietileno que producen aproximadamente $0.2 \mathrm{~m}^{3}$ de biogás $/ \mathrm{m}^{3}$ biodigestor día ${ }^{-1}$, dentro del rango psicrofílico, con biodigestores de $5 \mathrm{~m}^{3}$ es suficiente para cocinar 3-4 h diarias, sustituyendo los combustibles tradicionales. El coste de construcción de los biodigestores es 140.7 soles/ $\mathrm{m}^{3}$. A nivel financiero, la instalación es más viable cuando el biogás sustituye un combustible con valor de mercado como el gas propano, resultando en un payback de 2 años y 8 meses. Poggio et al, (2009) realizaron una investigación de adaptación de los biodigestores tubulares de plástico a climas fríos donde concluye que la sustitución de combustibles tradicionales (leña y bosta) y/o fósiles por biogás conlleva beneficios tanto ambientales como sanitarios para los usuarios. El coste total de los materiales empleados para el digestor de PVC (1746 soles) es un 73\% superior con respecto al digestor de polietileno (1 008 soles). 
El objetivo de esta investigación es proponer un biodigestor tubular de polietileno para producir biogás a partir de paja de arroz y agua del río Utcubamba en la ciudad de Bagua Grande Amazonas. Para lo cual se calculó el biogás producido a partir de paja de arroz y agua del río Utcubamba de manera experimental, utilizando tres biodigestores. A partir de los datos obtenidos se propone un biodigestor tubular de polietileno, proyectando los resultados obtenidos para sustituir el consumo de leña de una familia promedio. Los biodigestores tubulares de régimen estacionario se construyeron con bolsas de HDPE (polietileno de alta densidad). El volumen de biogás producido acumulado utilizando paja de arroz mezclado con agua del río Utcubamba fue $541 \mathrm{ml}$. Teniendo en cuenta los resultados obtenidos se propone un biodigestor tubular de polietileno para producir biogás a partir de paja de arroz, considerando que en menos de 4 años se podrá recuperar la inversión inicial (1 174 soles), si se utiliza polietileno de 7,26 $\mathrm{m}$ de longitud y $1,5 \mathrm{~m}$ de ancho, que genere $7,69 \mathrm{~m}^{3}$ de biogás, para sustituir el uso de leña que requiere una familia promedio en la cocción de sus alimentos, contribuir a reducir la contaminación ambiental, y mejorar la calidad de vida de las personas de Bagua Grande - Amazonas.

\section{MATERIAL Y MÉTODOS}

\subsection{Población muestra y muestreo}

La población de los residuos agrícolas abandonados después de la cosecha de arroz (variedad INIA 509-La Esperanza) lo constituyó un terreno agrícola de 2 hectáreas, ubicado en el sector Gonchillo Bajo de la ciudad de Bagua Grande. Y el agua de la cuenca baja del río
Utcubamba. La muestra lo constituyó 6 Kilogramos de paja de arroz durante época húmeda (marzo) y 6 kilogramos de paja de arroz durante época seca (septiembre) y la muestra de 12 litros de agua se obtuvo de la cuenca baja del río Utcubamba. Se realizó un muestreo no probabilístico de tipo intencional.

\subsection{Diseño de investigación}

Diseño cuasi experimental - Diseño de grupos no equivalentes con grupo control:

\section{$\mathrm{O}_{1} \mathrm{X} \mathrm{O}_{2}$ \\ $\mathrm{O}_{3} \mathrm{O}_{4}$}

(Maza y Villanueva, 2011)

Donde:

O1 y $\mathbf{O}_{3}$ : observación inicial $(2 \mathrm{Kg}$ de residuos de arroz)

X: variable independiente (agua del río Utcubamba)

$\mathbf{O}_{2}$ y $\mathbf{O}_{4}$ : observación final (volumen de biogás).

\subsection{Material biológico}

Se utilizó residuos agrarios del cultivo de arroz (paja seca), de variedad INIA 509 - La Esperanza, de un predio agrícola, cercano a la Universidad Politécnica Amazónica de Bagua Grande. Agua del río Utcubamba de la ciudad de Bagua Grande.

\subsection{Métodos, técnicas e instrumentos}

\subsubsection{Métodos experimentales}

En el desarrollo de la presente investigación se evaluó el efecto del agua del río Utcubamba como estimulante, sobre la variable dependiente que fue el volumen de biogás a partir de paja de arroz. Se realizaron 2 tratamientos con 3 repeticiones por cada uno. Después de un tiempo de retención de 
35 días, se calculó el volumen de biogás producido. Se analizó los datos para obtener el volumen óptimo de producción de biogás y proponer un biodigestor tubular de polietileno para producir biogás a partir de paja de arroz y agua del río Utcubamba en la ciudad de Bagua Grande.

\subsubsection{Técnicas de recolección de datos}

Se utilizó la técnica de recolección de datos, de fichas técnicas de registro de información. Los análisis de las aguas fueron realizados en laboratorio de Agua y Suelos del Instituto para el Desarrollo Sustentable de Ceja de Selva (INDES $-\mathrm{CES})$.

\subsubsection{Instrumentos y equipos de recolección de datos}

Ficha técnica de registro de datos, manómetro de tubería de butirato flexible, transparente y resistente al astillamiento, de precisión de $0-1$ bar, balanza digital, GPS, carta de alcanza de resultados de laboratorio del INDES - CES.

\subsection{Procedimiento}

1.- Determinación de las variables

2.-Preparación de dos grupos idénticos

3.- Medición inicial de ambos grupos o pre test

4.-Aplicación del estímulo o variable independiente y construcción de los biodigestores experimentales

5.- Construcción de los biodigestores experimentales
6.- Realización de una nueva medición o post test (calcular del volumen de biogás)

7.- Propuesta de un biodigestor tubular de polietileno según necesidades de combustible

Según Baculima, \& Rocano (2015) un metro cúbico de biogás de $60 \%$ de $\mathrm{CH}_{4}+40 \%$ de $\mathrm{CO}_{2}$ equivale a $1,3 \mathrm{Kg}$ de madera, teniendo en cuenta que una familia promedio utiliza $10 \mathrm{Kg}$ de madera por día entonces se necesitaría $7,69 \mathrm{~m}^{3}$ de biogás. Tomando el valor de la tabla 7 , se generan $0,277 \mathrm{~m}^{3}$ de biogas por $\mathrm{Kg}$ de paja de arroz, entonces para producir esos 7,69 $\mathrm{m}^{3}$ de biogás, se calcula lo siguiente.

Carga de paja de arroz $=7,69 \mathrm{~m}^{3} /\left(0,277 \mathrm{~m}^{3} / \mathrm{Kg}\right)$

Se obtiene que son necesarios $27,76 \mathrm{~kg}$ de paja de arroz al día.

Volumen líquido, gaseoso y volumen total

$V L=$ tiempo de retención (35 días) $\times 112$ litros $=$ 3920 litros

El volumen líquido representa las tres cuartas partes del volumen total.

$\mathrm{VT}=$ volumen total 5227 litros $\left(5.227 \mathrm{~m}^{3}\right)$.

7.1. Dimensionamiento del biodigestor tubular de polietileno.

7.1.1. Ancho de rollo y radio de la manga

El perímetro de la circunferencia viene dado por:

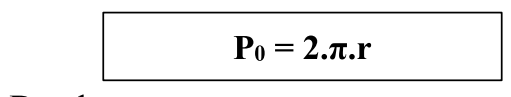

Donde:

$\mathrm{P}_{0}=$ Perímetro de la circunferencia 
$\pi(\mathrm{pi})=3,1416$

$\mathrm{r}=$ radio de la circunferencia

\section{$r=0.48$ metros}

$\mathrm{r}=\mathrm{P}_{0} / 2 . \pi$

Volumen y longitud del biodigestor tubular de polietileno

$$
\mathrm{V}_{\text {cilindro }}=\mathbf{p i} \times \mathbf{r}^{2} \times \mathbf{L}
$$

Donde:

$\mathrm{V}_{\text {cilindro }}=5.227 \mathrm{~m}^{3}$

pi $=3.1416$

$\mathrm{r}=0,48$ metros

$\mathrm{L}=$ longitud del biodigestor

\section{$\mathbf{L}=7,26$ metros}

\section{RESULTADOS}

\subsection{Análisis de datos:}

Tabla 1: Informe de estadísticos: Volúmenes de biogás en época húmeda y época seca

\begin{tabular}{|c|c|c|c|c|}
\hline \multicolumn{5}{|c|}{$\begin{array}{c}\text { Descriptivos } \\
\text { Volumen de biogás }\end{array}$} \\
\hline & $\mathrm{N}$ & Media & $\begin{array}{l}\text { Desviación } \\
\text { estándar }\end{array}$ & $\begin{array}{l}\text { Error } \\
\text { estándar }\end{array}$ \\
\hline $\begin{array}{l}\text { A.R.U, } \\
\text { E.H, } \\
\text { A.R.U, } \\
\text { E.S. } \\
\text { Total }\end{array}$ & 3 & $\begin{array}{r}534400 \\
.541233 \\
, 537817\end{array}$ &, 00012124 & $\begin{array}{l}, 0007000 \\
, 0004667 \\
\mathbf{0 0 1 5 7 3 6}\end{array}$ \\
\hline
\end{tabular}

A.R.U, E.H: Agua del río Utcubamba época húmeda Temperatura $\quad(26,97$

A.R.U, E.S: Agua del río Utcubamba, época seca. Temperatura $\left(27,71^{\circ} \mathrm{C}\right)$

De la tabla 1, se deduce que el T2: agua de río Utcubamba en época seca + paja de arroz, arrojó el mayor valor equivalente a $\mathbf{0 , 5 4 1} \mathrm{ml}$ de biogás y la media del T1: agua de río en época húmeda + paja de arroz es $\mathbf{0 , 5 3 4} \mathrm{ml}$ de biogás.

\section{Volumen de biogás producido en diferentes} temperaturas

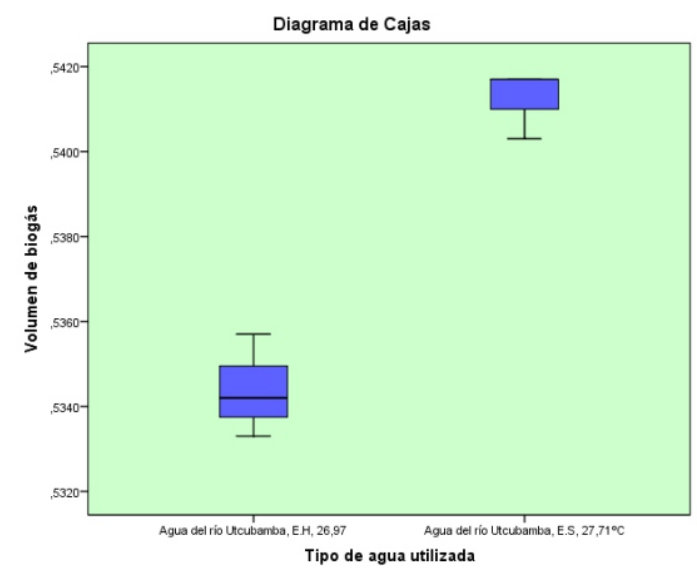

Figura 1: Diagrama de Cajas (Volumen de biogás por temperatura).

En la figura 1 los dos tratamientos difieren entre sí. El T2 (agua del río Utcubamba en época seca) genera el mayor volumen de biogás y el T1 (agua del río Utcubamba en época húmeda) genera el menor volumen de biogás a partir de paja de arroz.

\subsection{Propuesta del biodigestor tubular de polietileno según las necesidades de combustible para una familia promedio.}

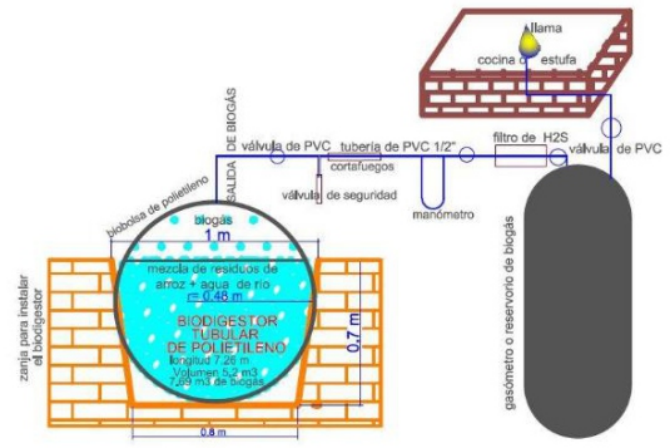

Figura 2: Diseño del biodigestor tubular de polietileno. 
Corte transversal del biodigestor tubular de polietileno propuesto, la zanja donde se ubicará tiene las paredes inclinadas (base superior de $1 \mathrm{~m}$, base inferior de $0,8 \mathrm{~m}$, y una altura de $0,7 \mathrm{~m})$. El biodigestor está diseñado para producir 7,69 $\mathrm{m}^{3} \mathrm{de}$ biogás, con una carga inicial de sustrato de $28 \mathrm{Kg}$, un volumen de agua de 84 litros, volumen liquido de $3,920 \mathrm{~m}^{3}$, volumen gaseoso de $1,307 \mathrm{~m}^{3}$, siendo el volumen total $5,2 \mathrm{~m}^{3}$, con un radio de $0,48 \mathrm{~m}$ y una longitud de 7,26 m. Para lo cual se utilizará un rollo de polietileno de $1,5 \mathrm{~m}$ de ancho y 8,26 $\mathrm{m}$ de largo, que opere a una temperatura promedio de $20-35^{\circ} \mathrm{C}$, durante un tiempo de retención de 35 días.

\subsection{Evaluación económica del biodigestor tubular de polietileno propuesto}

Tabla 2: Costo total del biodigestor tubular de polietileno propuesto.

\begin{tabular}{lc}
\hline \multicolumn{1}{c}{ Descripción } & Costo \\
\hline Construcción del biodigestor & 586.00 \\
Herramientas para instalación & 298.90
\end{tabular}

Mano de obra para construcción e $\quad 290.00$ instalación del biodigestor

\section{Total}

1174.90

De la tabla 2 se deduce que el costo total para la construcción del biodigestor tubular de polietileno para producir biogás a partir de la paja de arroz es $1 \quad 174.90$ soles, los cuales incluyen la construcción, herramientas, mano de obra e instalación.

Tabla 3: Beneficios del uso del biodigestor tubular de polietileno en un periodo de 5 años

\begin{tabular}{lccc}
\hline \multirow{2}{*}{ Beneficios } & \multicolumn{3}{c}{ Años } \\
\cline { 2 - 4 } V.B. (soles) & $\mathbf{1}$ & $\mathbf{2}$ & $\mathbf{5}$ \\
*Otros costos & 365.0 & 365.0 & 365.00 \\
Mano de obra & 884,9 & 0.00 & 0.00 \\
& & & \\
Costos totales (soles) & 290.0 & 0.00 & 0.00 \\
$\begin{array}{l}\text { Beneficios netos por } \\
\text { año (soles) }\end{array}$ & -1174.9 & -809.9 & 285.10 \\
& -809.9 & $-444,9$ & 650.10 \\
\hline
\end{tabular}

V.B: Valor del biogás

*Otros costos: (materiales y herramientas).

En términos económicos los beneficios que presenta la utilización del biodigestor significa un ahorro en el primer año de s/. 365.00 para una producción de 7,69 $\mathrm{m}^{3}$ de biogás por día.

\section{DISCUSIÓN}

Contreras et al (2012) "adicionaron inóculos provenientes de lodos anaerobios de plantas de biogás, realizaron su experimento en condiciones termofílicas $55^{\circ} \mathrm{C}$ y en condiciones mesofílicas $37^{\circ} \mathrm{C}$, obteniendo $0,50 \mathrm{~m}^{3} \mathrm{kgSV}^{-1}$ y $0,46 \mathrm{~m}^{3} \mathrm{kgSV}^{-}$ ${ }^{1}$ respectivamente, a partir de paja de arroz como monosustrato". En la presente investigación el mayor volumen de biogás producido es $0,28 \mathrm{~m}^{3} \mathrm{de}$ biogás por $\mathrm{Kg}$ de paja de arroz bajo condiciones mesofílicas $\left(27,71^{\circ} \mathrm{C}\right)$ durante época seca y durante época húmeda $\left(26,97^{\circ} \mathrm{C}\right)$ se obtuvo $0,27 \mathrm{~m}^{3} / \mathrm{Kg}$. Por lo tanto, se evidencia que la temperatura influyó de forma favorable en la producción de biogás al acelerar el proceso de biodigestión anaeróbica, además Contreras et al 2012 obtuvieron mayor volumen de biogás probablemente porque adicionaron inóculos provenientes de lodos anaerobios de plantas de biogás, lo cual estimula la producción de biogás, además realizaron su experimento a mayor 
temperatura lo que también estimula una mayor producción de biogás.

Ferrer et al., (2009), “instalaron biodigestores piloto que producen aproximadamente $0.2 \mathrm{~m}^{3} \mathrm{de}$ biogás $/ \mathrm{m}^{3}$ biodigestor día ${ }^{-1}$, dentro del rango psicrofílico, con biodigestores de $5 \mathrm{~m}^{3}$ es suficiente para cocinar 3-4 h diarias, sustituyendo los combustibles tradicionales". El biodigestor propuesto en esta investigación es de 7,26 m y está diseñado para producir $7,69 \mathrm{~m}^{3}$ de biogás, con una carga inicial de sustrato de $28 \mathrm{Kg}$ de paja de arroz y 84 litros de agua. El biodigestor que se propone es de mayor longitud que el de Ferrer et al, considerando que al utilizar paja de arroz se genera menor cantidad de biogás que al utilizar el sustrato que utilizó Ferrer et al.

El coste de construcción de los biodigestores de Ferrer et al, (2009) es 140.7 soles/ m³ . Mientras que es esta investigación se propone un biodigestor tubular de polietileno de $5.227 \mathrm{~m}^{3}$ para producir biogás a partir de paja de arroz para generar 7,69 $\mathrm{m}^{3}$ de biogás. El coste de construcción del biodigestor es 1174.90 soles, 152.7 soles $/ \mathrm{m}^{3}, 12$ soles más que el costo por metro cubico obtenido por Ferrer et al, (2009). Según Poggio et al, (2006) el coste total de los materiales empleaos para el digestor de PVC (1746 soles). Este valor es un $73 \%$ superior con respecto al digestor de polietileno que se propone en esta investigación (1 008 soles). Se estimó un costo total para la construcción de un biodigestor tubular de $5.227 \mathrm{~m}^{3}$ es 1174.9 soles, 166.9 soles más que el costo estimado por Poggio et al, (2006).

\section{CONCLUSIONES}

Las dimensiones óptimas del biodigestor tubular de polietileno son 7, $26 \mathrm{~m}$ de longitud y 1,5 $\mathrm{m}$ de ancho, que generaría 7,69 $\mathrm{m}^{3}$ de biogás diariamente para producir biogás a partir de paja de arroz y agua del río Utcubamba en la ciudad de Bagua Grande.

Con la instalación de los biodigestores experimentales se determinó que el volumen promedio de biogás generado a partir de paja de arroz y agua del río Utcubamba es $(0,269 \mathrm{ml}$ de biogás/ Kg de paja de arroz), obteniéndose el valor óptimo $(0,541 \mathrm{ml}$ de biogás $/ 2 \mathrm{Kg}$ de paja de arroz o 0,2706 ml de biogás/ Kg de paja de arroz) en época seca a $27,71^{\circ} \mathrm{C}$.

Un biodigestor tubular de polietileno de 7,26 $\mathrm{m}$ de largo para producir que genere diariamente 7,69 $\mathrm{m}^{3}$ de biogás a partir de paja de arroz, es una propuesta viable considerando que en menos de 4 años se podrá recuperar la inversión inicial (1 174 soles), para sustituir la leña que emplea una familia promedio en la cocción de alimentos, reducir la contaminación ambiental, y mejorar la calidad de vida de las personas en la ciudad de Bagua Grande - Amazonas.

\section{REFERENCIA BIBLIOGRÁFICA}

Baculima, M. V., \& Rocano, G. C. (2015). Estudio para la determinación de la producción de energía eléctrica a partir del aprovechamiento del biogás de una granja porcina ubicada en la ciudad de Azogues. Tesis de pregrado, Universidad Politécnica Salesiana sede Cuenca, Ecuador.

Contreras, L. M., Pereda, I., \& Romero, O. (2012). Aprovechamiento energético de residuos arroceros por bio-conversión. Caso de estudio cuba. Revista DYNA Energía y Sostenibilidad, 1(1), 1-11. 
Ferrer, I., Uggetti, E., Poggio, D., Martí, J., \& Velo, E. (2009). Producción de biogás a partir de residuos orgánicos en biodigestores de bajo coste. Perú.

García, A. (2010). Mejoramiento de la eficiencia energética y economica en el CAI Arrocero Sur del Jibaro mediante estudio de potenciales de ahorro por aprovechamiento enrgético de residuos. Tesis de Maestría en Eficiencia Energética, Centro Universitario José Martí Perez, Cuba.

Grupo Intergubernamental de Expertos sobre el Cambio Cimático (2007). Cambio climático 2007: Informe de síntesis (4). Recuperado el 20 de noviembre de 2015, desde https://www.ipcc.ch/pdf/assessmen t-report/ar4/syr/ar4_syr_sp.pdf

Gutiérrez, G., Moncada, I., Meza, M., Felix, A., Balderas, J., \& Gortáres, P. (2012). Biogás: una alternativa ecológica para la produccion de energía. Ide@s CONCITEG, 7 (85), 881-894.

Maza, C. y Villanueva, L. (2011). Diseño de investigación aplicado a las ciencias del medio ambiente. ( $1^{\mathrm{a}}$. ed.). Santiago de Chile: Universitaria

Poggio D, Ferrer I, Batet Ll y Velo, E (2009). Adaptación de biodigestores tubulares de plástico a climas fríos. Livestock Research for Rural Development. 21(152) Recuperado el 5 de mayo del 2016 desde: https://www.researchgate.net/profile/Ivet_ Ferrer/publication/287593627_Adaptation _of_plastic_tubular_biodigesters_to_cold _climates/links/572b2dd408ae2efbfdbdcf 51.pdf

Su, J., Hu, C., Yan, X., Jin, Y., Chen, Z., Guan, Q., Sun, C. (2015). Expression of barley
SUSIBA2 transcription factor yields highstarch low-methane rice. Nature, 53(1), 602-606. doi: 10.1038 / nature14673

Weiland, P. (2010), Biogas production: current state and perspectives. MINI-REVIEW'. Appl Microbiol Biotechnol, 1(85), 849860. 